\title{
Hyphomycetes decompositores do folhedo de Tibouchina pulchra Cogn.
}

\author{
ROSELY A.P. GRANDI ${ }^{1,3}$ e LUÍS F.P. GUSMÃO²
}

(recebido: 15 de agosto de 2001; aceito: 11 de janeiro de 2002)

\begin{abstract}
Decomposing Hyphomycetes on leaf litter of Tibouchina pulchra Cogn.). Twenty-two Hyphomycetes were obtained during a survey of mitosporic fungi associated with leaf litter of Tibouchina pulchra Cogn. in an Atlantic rain forest area at the "Reserva Biológica de Paranapiacaba", Santo André, São Paulo state, Brazil. The leaf litter was prepared by the washing technique with serial changes of sterile distilled water and incubated in moist-chambers. Rhinocladiella cristaspora Matsushima, Venustusynnema ciliata (Castañeda, G. Arnold \& A. Guerra) Castañeda \& Kendrick and Vermiculariopsiella cubensis (Castañeda) Nawawi, Kuthubutheen \& Sutton, are new records for Brazil; for these species, descriptions, geographical distribution, remarks and illustrations are provided.
\end{abstract}

RESUMO - (Hyphomycetes decompositores do folhedo de Tibouchina pulchra Cogn.). Durante o levantamento de fungos mitospóricos associados ao folhedo de Tibouchina pulchra Cogn. coletado na Reserva Biológica de Paranapiacaba (Mata Atlântica), Santo André, estado de São Paulo, vinte e dois Hyphomycetes foram isolados. As folhas passaram pela técnica da lavagem sucessiva de substratos com água destilada esterilizada e foram incubadas em câmaras-úmidas. Três espécies constituem novos registros para o Brasil, Rhinocladiella cristaspora Matsushima, Venustusynnema ciliata (Castañeda, G. Arnold \& A. Guerra) Castañeda \& Kendrick e Vermiculariopsiella cubensis (Castañeda) Nawawi, Kuthubutheen \& Sutton; para essas são apresentadas descrições, distribuição geográfica, comentários e ilustrações.

Key words - Mitosporic Fungi, biodiversity, decomposition, Atlantic rain forest

\section{Introdução}

O folhedo representa habitat peculiar para os fungos e é colonizado por inúmeras espécies atuantes no processo de decomposição. Os fungos mitospóricos destacam-se nesse processo, sendo os Hyphomycetes, muitas vezes, referidos como os principais colonizadores do folhedo (Dix \& Webster 1995).

Trabalhos sobre levantamento e caracterização taxonômica de Hyphomycetes decompositores de folhas de diferentes espécies vegetais ainda são poucos no Brasil. Provavelmente, Cedrela fissilis Vell. e Alchornea triplinervia (Spreng.) Müll. Arg. sejam as plantas mais bem estudadas quanto a presença desses fungos em suas folhas.

O folhedo de $C$. fissilis foi investigado a partir de coletas no Horto Florestal "Dr. Luiz Teixeira Mendes", município de Maringá, PR e na Reserva Florestal da Cidade Universitária "Armando de Salles Oliveira", município de São Paulo, SP. Assim, Gusmão et al. (1995) listaram 13 Hyphomycetes identificados apenas em nível

1. Instituto de Botânica, Caixa Postal 4005, 01061-970 São Paulo, SP, Brasil.

2. Universidade Estadual de Feira de Santana, Departamento de Ciências Biológicas, Caixa Postal 252, 44031-460 Feira de Santana, BA, Brasil.

3. Autor para correspondência: rapgrandi@sti.com.br genérico; Grandi et al. (1995) caracterizaram 19 táxons, sendo quatro citados pela primeira vez para o Brasil; Grandi \& Gusmão (1995) discutiram o gênero Gyrothrix, com três espécies; Gusmão \& Grandi (1996) apresentaram cinco espécies do grupo Beltrania e Gusmão \& Grandi (1997) relataram os Hyphomycetes que exibem conidióforos agrupados.

Com relação a $A$. triplinervia, destacam-se os estudos feitos com coletas de folhedo dessa planta provenientes da Reserva Biológica de Paranapiacaba, município de Santo André e da Reserva Ecológica de Juréia-Itatins, município de Peruíbe, ambas no estado de São Paulo. Dessa forma, para a Reserva Biológica de Paranapiacaba, Grandi (1994) apresentou alguns táxons e discutiu a distribuição geográfica do grupo como um todo; Grandi (1998) descreveu 19 táxons e ilustrou os que não possuíam ilustrações na literatura brasileira e Schoenlein-Crusius \& Milanez (1998a, b) listaram os fungos participantes do processo de sucessão nas folhas de $A$. triplinervia, colocadas no solo e na água. Ainda, Grandi \& Attili (1996) caracterizaram 16 espécies de Hyphomycetes decompositores, duas das quais como primeira citação para o Brasil, sendo este o único trabalho com material proveniente da Reserva de Juréia-Itatins.

$\mathrm{O}$ folhedo de Euterpe edulis Mart. e Miconia cabussu Hoehne foi também analisado quanto aos Hyphomycetes decompositores revelando, respectivamente, 26 e 55 táxons (Grandi 1999, Gusmão et al. 2001) incluindo uma espécie nova (Gusmão et al. 2000), todos 
provenientes da Reserva Biológica de Paranapiacaba.

Tibouchina pulchra Cogn. (Melastomataceae) é espécie arbórea e nativa, muito freqüente na Reserva Biológica de Paranapiacaba, ocorrendo na Serra do Mar, nos domínios da Mata Atlântica, incluindo regiões afetadas pela poluição aérea. Por ser espécie considerada indicadora de poluição, vem sendo utilizada em muitas pesquisas visando esclarecer as alterações provocadas por compostos poluentes em seu comportamento fisiológico e anatômico (Domingos 1998, Domingos et al. 1998, Furlan et al. 1999, Klumpp et al . $2000 \mathrm{a}, \mathrm{b}$, Moraes et al. $2000 \mathrm{a}, \mathrm{b}$ ).

Quanto a presença de fungos associados à $T$. pulchra, apenas o trabalho de Trufem \& Malatinszky (1995) aborda levantamento de micorrizas arbusculares na rizosfera dessa planta, com predominância de espécies de Acaulospora.

O presente trabalho teve por objetivos relatar os Hyphomycetes que ocorrem no folhedo de T. pulchra, agindo como decompositores, e caracterizar as espécies referidas pela primeira vez para o Brasil.

\section{Material e métodos}

Coletas bimestrais do folhedo de Tibouchina pulchra Cogn. foram efetuadas, entre outubro de 1995 e outubro de 1996, na Reserva Biológica de Paranapiacaba, município de

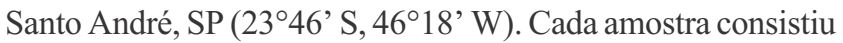
de 20 folhas em decomposição de um indivíduo de T. pulchra, sendo realizadas sete viagens para coleta. Seis amostras foram recolhidas por viagem, totalizando, assim, 42 amostras de folhedo.

Cada amostra de folhedo passou pela técnica da lavagem sucessiva de substratos, por 15 vezes cada uma, conforme descrição em Grandi \& Gusmão (1998). Esta técnica, em seus passos principais, consiste em lavar as folhas em decomposição com água destilada esterilizada, fragmentar esse substrato em pedaços menores $(5$ a $10 \mathrm{~mm}$ de comprimento) e colocá-los em duas câmaras-úmidas (placas de Petri com $9 \mathrm{~cm}$ de diâmetro e papel de filtro dentro). As câmaras são mantidas úmidas com adição de gotas de água destilada esterilizada, quando necessário, e observadas durante 45 dias, período em que os fungos são isolados.

Os Hyphomycetes foram isolados sob estereomicroscópio, colocados diretamente entre lâmina e lamínula com meios de montagem PVL (álcool polivinílico + ácido lático + fenol) ou PVL-lactofenol-azul-de-algodão, constituindo lâminas permanentes. Medidas das estruturas de importância taxonômica, para cada espécie, foram efetuadas 30 vezes, ou tantas vezes quanto possível para espécimes com pouca ocorrência. A identificação, sob microscópio, foi feita de acordo com a taxonomia tradicional para o grupo. Lâminas representativas dos táxons isolados foram incorporadas ao Herbário
Científico "Maria Eneyda P. Kauffmann Fidalgo" (SP), do Instituto de Botânica. Somente foram descritas e ilustradas em detalhes as espécies referidas pela primeira vez para o Brasil; para as demais foram indicadas as descrições e ilustrações já publicadas, incluindo literatura brasileira. Para todas as espécies foram apresentados breves comentários, incluindo as características diagnósticas e comparação com as espécies taxonomicamente mais próximas e a distribuição geográfica mundial.

\section{Resultados e Discussão}

Durante o período de estudo foram encontrados os seguintes táxons:

Beltrania malaiensis Wakefield, Kew Bull. 4:204. 1931.

Descrições e ilustrações: Pirozynski (1963), Gusmão et al. (2001).

Material examinado - BRASIL: São PAULO: Santo André, Reserva Biológica de Paranapiacaba, 14-X-1996, R.A.P. Grandi s.n. (SP 307611).

Difere de B. rhombica pelo maior tamanho dos conídios e dos apêndices, bem como pela cicatriz basal dos conídios, que são mais proeminentes em $B$. malaiensis. Não foram observadas setas neste material, estrutura de ocorrência infreqüente, como apontado por Pirozynski (1963). Espécie descrita pela primeira vez para o Brasil por Gusmão et al. (2001). Sua distribuição restringe-se ao Brasil, Índia e Malásia.

Beltrania rhombica Penzig, Nuovo G. Bot. Ital. 14:72. 1882.

Descrições e ilustrações: Pirozynski (1963), Gusmão \& Grandi (1996).

Material examinado - BRASIL: São PAUlo: Santo André, Reserva Biológica de Paranapiacaba, 10-VI-1996, R.A.P. Grandi s.n. (SP 307612).

É a espécie do gênero que ocorre com maior freqüência no folhedo. Produz setas em grande quantidade e tem a banda mediana dos conídios mais perceptível.

Espécie com distribuição ampla, em regiões tropicais a temperadas.

Beltraniella portoricensis (F.L. Stevens) Pirozynski \& Patil, Can. J. Bot. 48:575. 1970.

Descrições e ilustrações: Pirozynski (1963), Gusmão \& Grandi (1996).

Material examinado - BRASIL: São PAUlo: Santo André, Reserva Biológica de Paranapiacaba, 12-VIII-1996, R.A.P. Grandi s.n. (SP 307613).

Espécie comum no folhedo de várias plantas ocorrendo em regiões tropicais, subtropicais e temperadas. 
Chalara alabamensis Morgan-Jones \& Ingram, Mycotaxon 4:489. 1976.

Descrições e ilustrações: Morgan-Jones \& Ingram (1976), Grandi \& Gusmão (1996), Grandi (1998).

Material examinado - BRASIL: São PAULo: Santo André, Reserva Biológica de Paranapiacaba, 16-X-1995, R.A.P. Grandi s.n. (SP 307614).

C. alabamensis tem conidióforo ausente ou reduzido a apenas uma célula e fiálides grandes. A distribuição conhecida é restrita a quatro países (Brasil, Costa do Marfim, Estados Unidos da América e México) ocorrendo em vários substratos de plantas em decomposição, inclusive raízes.

Chalara hughesii Nag Raj \& Kendrick, N. Z. J. Bot. 12:118. 1974.

Descrições e ilustrações: Nag Raj \& Kendrick (1975), Kirk (1982a), Holubová-Jechová (1984).

Material examinado - BRASIL: São PAULO: Santo André, Reserva Biológica de Paranapiacaba, 16-X-1995, R.A.P. Grandi s.n. (SP 307615).

Difere de $C$. alabamensis por apresentar conidióforos definidos e septados. Espécie coletada em países de clima temperado (Argentina, Canadá, Checoslováquia, Estados Unidos da América, Japão, Nova Zelândia e Reino Unido), mas com referências para alguns tropicais (Brasil, Sri Lanka e Taiwan). Salienta-se que Kirk (1982a), ao estudar os fungos colonizadores de folhas em decomposição de Laurus nobilis L., na Inglaterra, analisou também essa espécie coletada no Brasil e depositada no herbário IMI, sugerindo ampla distribuição deste táxon.

Chloridium transvaalense Morgan-Jones, Sinclair \& Eicker, Mycotaxon 17:302. 1983.

Descrições e ilustrações: Morgan-Jones et al. (1983), Grandi (1998).

Material examinado - BRASIL: São PAulo: Santo André, Reserva Biológica de Paranapiacaba, 5-II-1996, R.A.P. Grandi s.n. (SP 307616).

Espécie referida pela primeira vez para o Brasil por Grandi (1994) e caracterizada taxonomicamente em Grandi (1998). Sua distribuição conhecida limita-se a África do Sul e Brasil.

Cladosporium oxysporum Berkeley \& Curtis, J. Linn. Soc. Lond. Bot. 10:362. 1868.

Descrições e ilustrações: McKemy \& Morgan-Jones (1991), Grandi (1998).

Material examinado - BRASIL: São PAULo: Santo André, Reserva Biológica de Paranapiacaba, 12-VIII-1996, R.A.P. Grandi s.n. (SP 307617).
Espécie muito comum nos trópicos e subtrópicos; no estado de São Paulo tem sido encontrada no folhedo de muitas plantas, indicando pouca especificidade.

Cryptophiale kakombensis Pirozynski, Can. J. Bot. 46:1124. 1968.

Descrições e ilustrações: Pirozynski (1968), Grandi \& Attili (1996).

Material examinado - BRASIL: São PAulo: Santo André, Reserva Biológica de Paranapiacaba, 10-VI-1996, R.A.P. Grandi s.n. (SP 307618).

Ápice do conidióforo setiforme pontiagudo e indiviso caracterizam o táxon. É uma das espécies mais comuns do gênero, encontrada na serapilheira de regiões tropicais e subtropicais.

Cryptophiale udagawae Pirozynski \& Ichinoe, Can. J. Bot. 46:1126. 1968.

Descrições e ilustrações: Pirozynski (1968), Grandi \& Attili (1996), Grandi (1998).

Material examinado - BRASIL: São PaUlo: Santo André, Reserva Biológica de Paranapiacaba, 8-IV-1996, R.A.P. Grandi s.n. (SP 307619).

Distingüe-se de C. kakombensis pelo ápice do conidióforo setiforme que exibe 1-3 ramificações dicotômicas. À semelhança de C. kakombensis, ocorre no folhedo, principalmente nos trópicos e subtrópicos.

Cylindrocladium scoparium Morgan, Bot. Gaz. 17:191. 1892.

Descrições e ilustrações: Grandi (1985), Grandi \& Attili (1996).

Material examinado - BRASIL: São PAULO: Santo André, Reserva Biológica de Paranapiacaba, 4-XII-1995, R.A.P. Grandi s.n. (SP 307620).

Apenas um exemplar apresentou vesícula, medindo $11,3 \times 4,8 \mu \mathrm{m}$, o que pode ocorrer freqüentemente (Peerally 1991). Com ampla distribuição, a espécie pode ser isolada de solo, serapilheira e é citada como fitopatógena de muitas plantas economicamente importantes para o Brasil (Peerally 1991).

Dictyochaeta novae-guineensis (Matsushima) Romero, Boln. Soc. argent. Bot. 22:76. 1983.

Descrições e ilustrações: Matsushima (1971), Grandi (1998).

Material examinado - BRASIL: São PAULO: Santo André, Reserva Biológica de Paranapiacaba, 8-IV-1996, R.A.P. Grandi s.n. (SP 307621).

Difere de D. simplex por apresentar setas cujo ápice pode se transformar em fiálide e assim produzir conídios. Sua distribuição é ainda restrita, apenas para 
Argentina, Brasil, Costa do Marfim, México e PapuaNova Guiné. Espécie referida pela primeira vez para o país por Grandi (1994) e descrita detalhadamente em Grandi (1998).

Dictyochaeta simplex (Hughes \& Kendrick) HolubováJechová., Folia Geobot. Phytotaxon. 19:434. 1984.

Descrições e ilustrações: Sutton \& Hodges Jr. (1975), Grandi \& Attili (1996), Grandi (1998).

Material examinado - BRASIL: São PaUlo: Santo André, Reserva Biológica de Paranapiacaba, 5-II-1996, R.A.P. Grandi s.n. (SP 307622).

Ausência de setas e conidióforos menores que em D. novae-guineensis caracterizam a espécie. Ocorre em regiões tropicais, subtropicais e temperadas.

Rhinocladiella cristaspora Matsushima, Microfungi of the Solomon Islands and Papua-New Guinea, Kobe, p. 49. 1971.

Figuras 1-2

Conidióforos eretos ou recurvados, espalhados, com poucos septos, sem ramificações, lisos, castanho-claros, 21,1-92,0 × 3,2-4,5 $\mu \mathrm{m}$. Células conidiogênicas integradas, apicais, cilíndricas, poliblásticas, com crescimento simpodial, denticuladas, lisas, castanho-claras. Conídios unicelulares, fusiforme-alargados ou lenticulares, extremidades afiladas, lisos, castanho-claros; base com cicatriz escura e ápice com apículo, muitos com conteúdo granular ou gutulados e uma linha longitudinal (fenda de germinação), 21,0-24,8 × 7,6-8,9 $\mu \mathrm{m}$.

Material examinado - BRASIL: São PAULo: Santo André, Reserva Biológica de Paranapiacaba, 16-X-1995, R.A.P. Grandi s.n. (SP 307623).

Distribuição geográfica: Cuba, Espanha (como Ardhachandra cristaspora (Matsushima) Subram. \& Sudha), Índia (como Ardhachandra cristaspora), Japão, Taiwan (como Ardhachandra cristaspora), Papua-Nova Guiné, Peru, Tanzânia (como Ardhachandra cristaspora).

Rhinocladiella foi proposto por Nannfeldt, em 1934, com a espécie-tipo $R$. atrovirens Nannf. (Hawksworth et al. 1995). Muitas espécies são pleomórficas, o que ocasionou a descrição de novos táxons dentro do gênero ou mesmo a criação de gêneros muito próximos. Hoje, Ardhachandra e Botrytoides são considerados seus sinônimos (Onofri \& Castagnola 1983, Hawksworth et al. 1995). R. cristaspora descrita por Pirozynski apresenta dois tipos de conídios, fusiformes e selenóides (Pirozynski 1972). No entanto, verificou-se, por microscopia eletrônica de varredura, que a espécie possui apenas um tipo, isto é, fusiformes ou lenticulares, com ou sem fenda de germinação, e assim concordante com a descrição original da espécie (Matsushima 1971, Onofri \& Castagnola 1983) e com os materiais obtidos. A espécie está sendo referida pela primeira vez para o Brasil.

Scolecobasidium variabile Barron \& Busch, Can. J. Bot. 40:83. 1962.

Descrições e ilustrações: Barron \& Busch (1962), Grandi (1985), Domsch et al. (1993).

Material examinado - BRASIL: São PAULo: Santo André, Reserva Biológica de Paranapiacaba, 8-IV-1996, R.A.P. Grandi s.n. (SP 307624).

Espécie muito comum nos solos e de distribuição ampla, isolada de solo de cerrado no estado de São Paulo por Grandi (1985). Domsch et al. (1993) referenciam-na como sinônimo de Scolecobasidium tshawytschae (Doty \& Slates) McGinnis \& Ajello, proposto em 1974. Os gêneros Heterosporium, Ochroconis e Scolecobasidium são muito próximos e várias espécies foram transferidas de um para outro sem haver, até o momento, tratamento taxonômico satisfatório. No presente trabalho optou-se por referir o táxon encontrado sob Scolecobasidium, gênero mais conhecido.

Speiropsis scopiformis Kuthubutheen \& Nawawi, Trans. Br. Mycol. Soc. 89:584. 1987.

Descrições e ilustrações: Mouchacca (1990), Gusmão et al. (2001).

Material examinado - BRASIL: São PAulo: Santo André, Reserva Biológica de Paranapiacaba, 10-VI-1996, R.A.P. Grandi s.n. (SP 307625).

Espécie caracterizada pela primeira vez para o Brasil por Gusmão et al. (2001) e até o momento com distribuição restrita, apenas para quatro países tropicais (Brasil, Cuba, Malásia e Nova Caledônia).

Sporidesmiella hyalosperma (Corda) P. M. Kirk var. hyalosperma, Trans. Br. Mycol. Soc. 79:481. 1982.

Descrições e ilustrações: Kirk (1982b), Grandi (1990, 1999).

Material examinado - BRASIL: São PAulo: Santo André, Reserva Biológica de Paranapiacaba, 4-XII-1995, R.A.P. Grandi s.n. (SP 307626).

Dentre as espécies do gênero, esta é a mais freqüentemente isolada, apresentando distribuição ampla e pouca especificidade.

Subulispora longirostrata Nawawi \& Kuthubutheen, Mycotaxon 30:459. 1987.

Descrições e ilustrações: Nawawi \& Kuthubutheen (1987), Gusmão et al. (2001). 

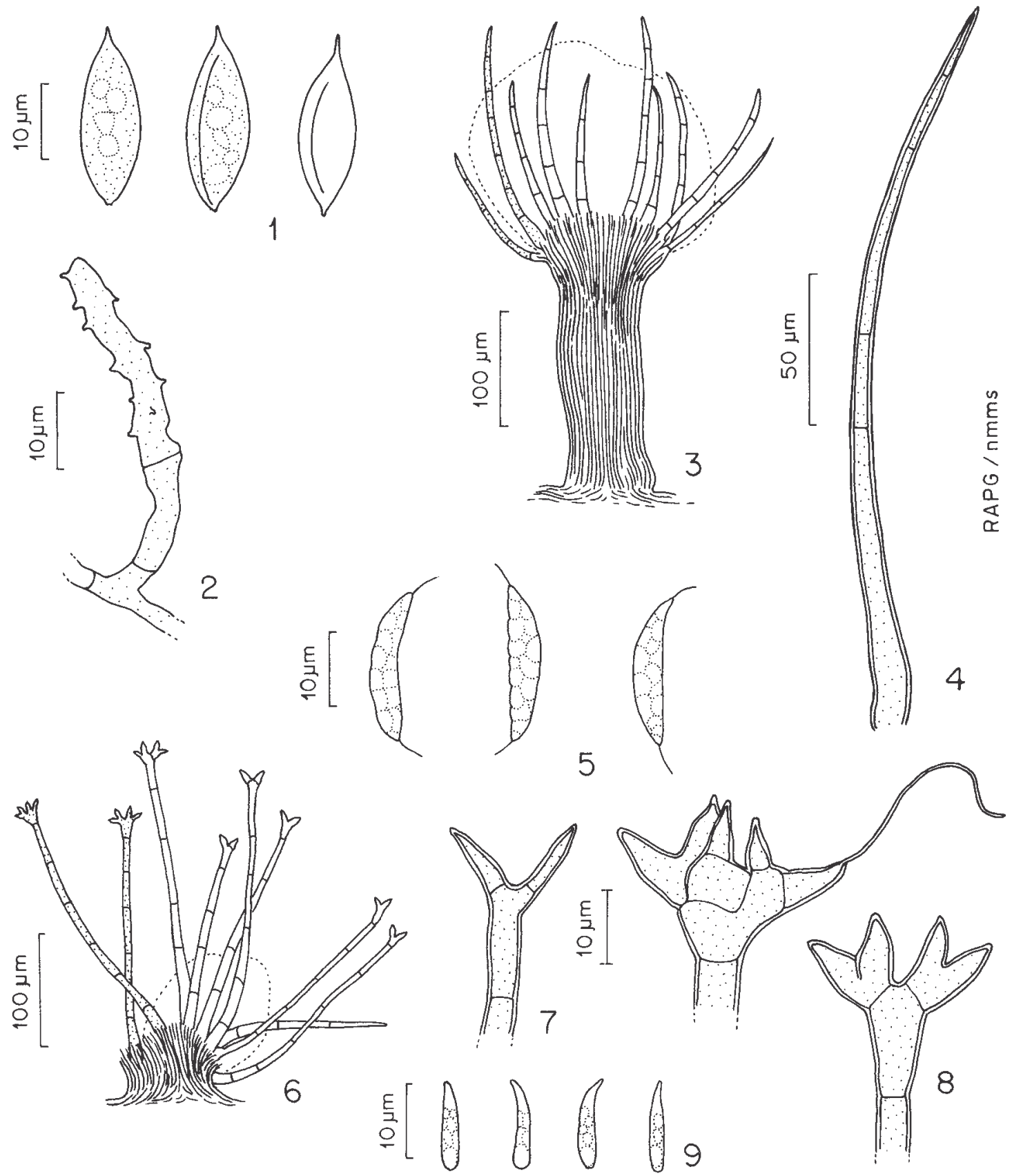

Figuras 1-9. 1-2. Rhinocladiella cristaspora. 1. Conídios. 2. Conidióforo com célula conidiogênica denticulada. 3-5. Venustusynnema ciliata. 3. Hábito do sinema. 4. Seta. 5. Conídios. 6-9. Vermiculariopsiella cubensis. 6. Hábito do esporodóquio. 7. Ápice de seta jovem, com apenas uma ramificação dicotômica. 8. Setas com ápices desenvolvidos, uma delas apresentando apêndice filiforme. 9. Conídios.

Material examinado - BRASIL: São PAUlo: Santo André, Reserva Biológica de Paranapiacaba, 16-X-1995, R.A.P. Grandi s.n. (SP 307545).

Conídios 3-septados e apêndices com característico arco na união com o conídio diferenciam esta espécie de $S$. procurvata. S. longirostrata foi reportada pela primeira vez para o Brasil por Gusmão et al. (2001) e tem distribuição muito restrita (Brasil e Malásia).
Subulispora procurvata Tubaki, Trans. Mycol. Soc. Japan 12:20. 1971.

Descrições e ilustrações: Tubaki \& Yokoyama (1971), Grandi (1998).

Material examinado - BRASIL: São PAulo: Santo André, Reserva Biológica de Paranapiacaba, 16-X-1995, R.A.P. Grandi S.n. (SP 307544); idem (SP 307627).

Conídios 2-3-septados (às vezes com septos pouco 
evidentes) maiores que em $S$. longirostrata e apêndices sem arco caracterizam a espécie, que é a mais bem distribuída do gênero, isolada em regiões tropicais a temperadas.

Thozetella cristata Pirozynski \& Hodges Jr., Can. J. Bot. 51:168. 1973.

Descrições e ilustrações: Pirozynski \& Hodges Jr. (1973), Gusmão \& Grandi (1997), Grandi (1998).

Material examinado - BRASIL: São PAUlo: Santo André, Reserva Biológica de Paranapiacaba, 12-VIII-1996, R.A.P. Grandi s.n. (SP 307628).

O gênero Thozetella é característico da serapilheira e esta espécie tem sido isolada a partir de folhas em decomposição de várias plantas. Espécie isolada no Brasil, Estados Unidos da América, Itália, Japão e México.

Venustusynnema ciliata (Castañeda, G. Arnold \& A. Guerra) Castañeda \& Kendrick, Univ. Waterloo Biol. Ser. 32:45. 1990.

Figuras 3-5.

Sinema ereto, cilíndrico, curto ou alongado, provido de setas características, castanho-claro ou castanho-amarelado, com conídios agrupados em mucilagem na região apical, 97-225 × 48,5-97 $\mu \mathrm{m}$. Setas numerosas, originadas aproximadamente no terço superior do sinema, recurvadas para o centro da estrutura, sem ramificações, geralmente com base alargada, ápice pontiagudo, septadas, lisas, parede espessa, castanho-amareladas a castanhas, 130-290 × 8-13 $\mu \mathrm{m}$. Conidióforos densamente unidos, de difícil individualização, castanho-claros; células conidiogênicas tipo fiálide, no ápice dos conidióforos, cilíndricas, densamente unidas, castanho-claras; região fértil em forma de leque. Conídios unicelulares, alantóides ou lunados, lisos, hialinos, caracteristicamente gutulados, com uma sétula filiforme e hialina em cada extremidade, às vezes pouco perceptíveis, $16,3-21 \times 3,5-4,3 \mu \mathrm{m}$. Conídios unidos em massa esbranquiçada e refringente, protegida pelas setas.

Material examinado - BRASIL: São PAULo: Santo André, Reserva Biológica de Paranapiacaba, 5-II-1996, R.A.P. Grandi s.n. (SP 307629).

Distribuição geográfica: Cuba.

As medidas dos conídios ora obtidas são maiores do que aquelas apresentadas por Castañeda Ruiz \& Kendrick (1990), isolada a partir de caule morto de Sorghum halepense (L.) Pers. (13-16 × 3-4 $\mu \mathrm{m})$. No entanto, concorda com as medidas obtidas para essas estruturas, quando cultivado em meio de cultura
(Castañeda Ruiz et al. 1983), como Saccardaea ciliata Castañela, G. Arnold \& A. Guerra. Acreditamos que essa espécie possa apresentar certas variações nas estruturas, quando isolada de diferentes substratos, como na quantidade de setas, morfologia do sinema, coloração e nas próprias medidas destacando-se, inclusive, a fase de desenvolvimento dos isolados. Portanto, consideramse essas diferenças obtidas como variação normal da espécie. Grandi \& Gusmão (1996) já haviam isolado Venustusynnema sp. a partir de raízes em decomposição de Calathea zebrina Lindl., proveniente da Mata Atlântica, em Paranapiacaba, SP que, à época, foi referenciada apenas em nível genérico. A comparação deste material com os obtidos no presente estudo revelou que trata-se, mesmo, de $V$. ciliata. Até o momento apenas esta espécie constitui o gênero, a qual está sendo reportada pela primeira vez para o país.

Vermiculariopsiella cubensis (Castañeda) Nawawi, Kuthubutheen \& Sutton, Mycotaxon 37:180. 1990.

Figuras 6-9.

Esporodóquio arredondado, circundado por setas e conídios agrupados em massa no interior do conidioma. Setas eretas, septadas, lisas, castanhas, solitárias ou mais comumente originadas em fascículos e recurvadas em direção à massa conidial, extremidades pontiagudas simples ou freqüentemente com ramificações dicotômicas curtas, 161,7-291,1 × 5,9-9,7 $\mu \mathrm{m}$ na base (comprimento incluindo as ramificações apicais). Ápice das setas com ramificações curtas, geralmente dicotômicas, que podem originar novas ramificações dicotômicas, terminando com células cônicas, sem septos ou com apenas um, lisas, castanho-claras. Células cônicas 5,5-13,1 $\mu \mathrm{m}$ compr. e algumas exibindo um apêndice filiforme e hialino. Conidióforos curtos, hialinos, densamente agrupados. Células conidiogênicas tipo fiálide, hialinas, no ápice dos conidióforos. Conidióforos e células conidiogênicas protegidos pelas setas e difíceis de serem observados individualizados. Conídios unicelulares, lisos, hialinos, cilíndricos, com uma das extremidades arredondada e outra ligeiramente curva e afilada, freqüentemente gutulados, 8,6-13,6 $\times 1,8-2,8 \mu \mathrm{m}$. Conídios unidos em mucilagem de coloração branca refringente.

Material examinado - BRASIL: São PAUlo: Santo André, Reserva Biológica de Paranapiacaba, 8-IV-1996, R.A.P. Grandi s.n. (SP 307630).

Distribuição geográfica: Cuba.

Apenas três espécies dentro do gênero exibem setas ramificadas e $V$. cubensis é a única que apresenta setas com várias ramificações dicotômicas apicais curtas 
e células terminais com apêndices filiformes hialinos. Atualmente dez espécies constituem o gênero, que tem Oramasia Urries, descrito em 1956, como sinônimo (Hawksworth et al. 1995). Todas as espécies conhecidas foram isoladas a partir de folhas ou ramos em decomposição. Somente V. immersa (Desm.) Bender já havia sido coletada no Brasil, referida pelo seu nome antigo Oramasia hirsuta Urries var. hirsuta, proveniente dos estados de Espírito Santo, Minas Gerais e Pernambuco (Sutton 1978). V. cubensis está sendo referida pela primeira vez para o Brasil.

Wiesneriomyces laurinus (Tassi) P.M. Kirk, Trans. Br. Mycol. Soc. 82:748. 1984.

Descrições e ilustrações: Kirk (1981), Gusmão \& Grandi (1997).

Material examinado - BRASIL: São PAUlo: Santo André, Reserva Biológica de Paranapiacaba, 14-X-1996, R.A.P. Grandi s.n. (SP 307631).

Espécie caracterizada pelas setas vigorosas castanhas e pontiagudas ao redor do esporodóquio e conídios unicelulares, hialinos, mas unidos em cadeias, geralmente com 5-7 elementos, dando a impressão de estrutura única; considerada cosmopolita.

Alguns dos fungos encontrados neste levantamento são colonizadores de folhedo, para outros, entretanto, há poucas informações. Assim, o grupo Beltrania, aqui representado por Beltrania malaiensis, B. rhombica e Beltraniella portoricensis, é característico da serapilheira, com várias espécies apresentando distribuição ampla (Pirozynski \& Patil 1970, Gusmão \& Grandi 1996). Situação semelhante é verificada para Cladosporium oxysporum, Cylindrocladium scoparium, Scolecobasidium variabile, Sporidesmiella hyalosperma var. hyalosperma, espécies de Subulispora, de Thozetella e Wiesneriomyces laurinus, os quais parecem ocorrer em vários substratos e demonstrando, portanto, pouca especificidade.

Espécies de Chalara têm sido referidas para países de clima temperado (Nag Raj \& Kendrick 1975) mas $C$. alabamensis tem sido encontrada em várias ocasiões (Grandi et al. 1995, Grandi \& Gusmão 1996, Grandi 1998, 1999, Gusmão et al. 2001); C. aurea (Cda.) Hughes sobre A. triplinervia (Grandi \& Attili 1996); C. cylindrosperma (Cda.) Hughes e C. microspora (Cda.) Hughes sobre Miconia cabussu (Gusmão et al. 2001) e $C$. hughesii no presente estudo. Da mesma forma, isto também ocorre com espécies de Chloridium e Dictyochaeta (à exceção apenas de D. simplex, que é comum), encontradas algumas vezes (Grandi et al. 1995, Grandi 1998, 1999, Gusmão et al. 2001). Assim, é possível que quanto mais substratos foliares forem analisados, maior probabilidade de que sejam encontradas espécies nunca antes relatadas para o Brasil ou mesmo para os trópicos, uma vez que nossas condições climáticas favorecem grandemente $o$ crescimento desses fungos.

Para os táxons Rhinocladiella cristaspora, Speiropsis scopiformis, Venustusynnema ciliata e Vermiculariopsiella cubensis as observações de ocorrência na serapilheira são recentes.

Trabalhos sobre levantamento de Hyphomycetes a partir de folhas em decomposição utilizando a técnica de lavagem e incubação em câmaras-úmidas têm revelado que, em média, $22,5 \%$ dos fungos obtidos são novas referências para o país, incluindo os dados ora obtidos (Grandi et al. 1995, Grandi \& Attili 1996, Grandi \& Gusmão 1996, Grandi 1998, 1999, Gusmão et al. 2001). Embora os locais, épocas de coleta e os substratos nem sempre sejam comparáveis, a técnica utilizada tem se mostrado eficiente para o conhecimento dos Hyphomycetes presentes no folhedo, uma vez que permite o crescimento dos fungos ativos no interior das folhas em decomposição (Harley \& Waid 1955). Dada a riqueza em espécies vegetais do nosso país e as poucas plantas cujos Hyphomycetes colonizadores foram inventariados, podemos inferir que inúmeros fungos ainda serão descritos como novos e que tantos outros terão sua ocorrência detectada na serapilheira.

\section{Referências bibliográficas}

BARRON, G.L. \& BUSCH, L.V. 1962. Studies on the soil Hyphomycete Scolecobasidium. Canadian Journal of Botany 40:77-84.

CASTAÑEDA RUIZ, R.F. \& KENDRICK, B. 1990. Conidial Fungi from Cuba: I. University of Waterloo Biology Series 32:1-53.

CASTAÑEDA RUIZ, R.F., GUERRA, A.G. \& ARNOLD, G.R.W. 1983. Nueva especie del género Saccardaea Cavara: $S$. ciliata Castañeda, G. Arnold et A. Guerra sp. nov. Revista del Jardín Botánico Nacional 4:27-34.

DIX, N.J. \& WEBSTER, J.W. 1995. Fungal ecology. Chapman $\&$ Hall, London.

DOMINGOS, M. 1998. Biomonitoramento da fitotoxicidade da poluição aérea e da contaminação do solo na região do complexo industrial de Cubatão, São Paulo, utilizando Tibouchina pulchra Cogn. como espécie indicadora. Tese de doutorado, Universidade de São Paulo, São Paulo. 
DOMINGOS, M., KLUMPP, A. \& KLUMPP, G. 1998. Air pollution impact on the Atlantic forest in the Cubatão region, SP, Brazil. Ciência e Cultura 50:230-236.

DOMSCH, K.H., GAMS, W. \& ANDERSON, T.-H. 1993. Compendium of Soil Fungi. v.1, IWH-Verlag, Eching.

FURLAN, C.M., SALATINO, A. \& DOMINGOS, M. 1999. Leaf contents of nitrogen and phenolic compounds and their bearing with the herbivore damage to Tibouchina pulchra Cogn. (Melastomataceae), under the influence of air pollutants from industries of Cubatão, São Paulo. Revista Brasileira de Botânica 22:317-323.

GRANDI, R.A.P. 1985. Hyphomycetes do estado de São Paulo. 1. Espécies do cerrado da Reserva Biológica de MojiGuaçu. Rickia 12:125-145.

GRANDI, R.A.P. 1990. Hyphomycetes decompositores 1. Espécies associadas às raízes de Calathea stromata (horticultural). Revista Brasileira de Biologia 50:123-132.

GRANDI, R.A.P. 1994. Hyphomycetes sobre folhas em decomposição de Alchornea triplinervia (Spreng.) M. Arg. provenientes da Reserva Biológica do Alto da Serra de Paranapiacaba, Santo André, SP (Mata Altântica). In Anais do III Simpósio de Ecossistemas da Costa Brasileira (S. Watanabe, coord.). ACIESP, São Paulo, v.2, p.118-124.

GRANDI, R.A.P. 1998. Hyphomycetes decompositores do folhedo de Alchornea triplinervia (Spreng.) Müll. Arg. Hoehnea 25:133-148.

GRANDI, R.A.P. 1999. Hifomicetos decompositores do folhedo de Euterpe edulis Mart. Hoehnea 26:87-101.

GRANDI, R.A.P. \& ATTILI, D.S. 1996. Hyphomycetes on Alchornea triplinervia (Spreng.) Müell. Arg. leaf litter from the Ecological Reserve Juréia-Itatins, State of São Paulo, Brazil. Mycotaxon 60:273-386.

GRANDI, R.A.P., GRANDI, A.C. \& DELITTI, W.B.C. 1995. Hyphomycetes sobre folhas em decomposição de Cedrela fissilis Vell. Hoehnea 22:27-37.

GRANDI, R.A.P. \& GUSMÃO, L.F.P. 1995. Espécies de Gyrothrix (Hyphomycetees) no folhedo de Cedrela fissilis Vell., em Maringá, PR, Brasil. Hoehnea 22:191-196.

GRANDI, R.A.P. \& GUSMÃO, L.F.P. 1996. Hyphomycetes decompositores de raízes de Calathea zebrina (Sims) Lindl. (Marantaceae), provenientes da Reserva Biológica do Alto da Serra de Paranapiacaba, Santo André, SP, Brasil. Revista Brasileira de Botânica 19:165-172.

GRANDI, R.A.P. \& GUSMÃO, L.F.P. 1998. A técnica da lavagem sucessiva de substratos de plantas como subsídio para estudos da associação fungo/substrato e diversidade de Hyphomycetes nos ecossistemas. In Anais do IV Simpósio de Ecossistemas Brasileiros (S. Watanabe, coord.). ACIESP, São Paulo, v.2, p.80-90.

GUSMÃO, L.F.P. \& GRANDI, R.A.P. 1996. Espécies do grupo Beltrania (Hyphomycetes) associadas a folhas de Cedrela fissilis Vell. (Meliaceae), em Maringá, PR, Brasil. Hoehnea 23:91-102.
GUSMÃO, L.F.P. \& GRANDI, R.A.P. 1997. Hyphomycetes com conidioma dos tipos esporodóquio e sinema associados a folhas de Cedrela fissilis (Meliaceae), em Maringá, PR, Brasil. Acta Botanica Brasilica 11:123-134.

GUSMÃO, L.F.P., GRANDI, R.A.P. \& MILANEZ, A.I. 2000. A new species of Beltraniopsis from Brazil, with a key to the known species. Mycological Research 104:251-253.

GUSMÃO, L.F.P., GRANDI, R.A.P. \& MILANEZ, A.I. 2001. Hyphomycetes from leaf litter of Miconia cabussu in the Brazilian Atlantic rain forest. Mycotaxon 79:201-213.

GUSMÃO, L.F.P., SCHWAN-ESTRADA, K.R.F. \& SANTOS, V.D. 1995. Fungos associados à decomposição de folhas de Aspidosperma polyneuron Mull. Arg. e de Cedrela fissilis Vell. In Anais do IX Congresso da Sociedade Botânica de São Paulo (M.R.P., Noronha, coord.). SBSP, São Paulo, p.61-65.

HARLEY, J.L. \& WAID, J.S.1955. A method of studing active mycelia on living roots and other surfaces in the soil. Transactions of the British Mycological Society 38:104-118.

HAWKSWORTH, D.L., KIRK, P.M., SUTTON, B.C.\& PEGLER, D.N. 1995. Ainsworth \& Bisby's Dictionary of the Fungi. CAB International, Wallingford.

HOLUBOVÁ-JECHOVÁ, V. 1984. Lignicolous Hyphomycetes from Czechoslovakia 7. Chalara, Exochalara, Fusichalara and Dictyochaeta. Folia Geobotanica et Phytotaxonomica 19:387-438.

KIRK, P.M. 1981. New or interesting microfungi III. A preliminary account of microfungi colonizing Laurus nobilis leaf litter. Transactions of the British Mycological Society 77:457-473.

KIRK, P.M. 1982a. New or interesting microfungi V. Microfungi colonizing Laurus nobilis leaf litter. Transactions of the British Mycological Society 78:293-303.

KIRK, P.M. 1982b. New or interesting microfungi VI. Sporidesmiella gen. nov. (Hyphomycetes). Transactions of the British Mycological Society 79:479-489.

KLUMPP, A., DOMINGOS, M. \& PIGNATA, M.L. 2000a. Air pollution and vegetation damage in South America State of knowledge and perspectives. In Environmental pollution and plant responses (S.B.Agrawal \& M. Agrawal, eds.). Lewis Publishers/CRC Press, New York, p.111-136.

KLUMPP, G., FURLAN, C.M., DOMINGOS, M. \& KLUMPP, A. 2000b. Response of stress indicators and growth parameters of Tibouchina pulchra Cogn. exposed to air and soil pollution near the industrial complex of Cubatão, Brazil. The Science of the Total Environment 246:79-91.

MATSUSHIMA, T. 1971. Microfungi of the Solomon Islands and Papua-New Guinea. Kobe.

MCKEMY, J.M. \& MORGAN-JONES, G. 1991. Studies in the genus Cladosporium sensu lato. IV. Concerning Cladosporium oxysporum, a plurivorous, predominantly saprophytic species in warm climates. Mycotaxon 41:297-405. 
MORAES, R.M., DELITTI, W.B.C. \& MORAES, J.A.P.V. 2000a. Fotossíntese líquida e crescimento de Tibouchina pulchra Cogn. nas proximidades de indústrias petroquímicas, em Cubatão, SP. Hoehnea 27:77-85.

MORAES, R.M., DELITTI, W.B.C. \& MORAES, J.A.P.V. 2000 b. Respostas de indivíduos jovens de Tibouchina pulchra Cogn. à poluição aérea de Cubatão, SP: fotossíntese líquida, crescimento e química foliar. Revista Brasileira de Botânica 23:441-447.

MORGAN-JONES, G. \& INGRAM, E.G. 1976. Notes on Hyphomycetes. XII. A new species of Chalara. Mycotaxon 4:489-492.

MORGAN-JONES, G., SINCLAIR, R.C.\& EICKER, A. 1983. Notes on Hyphomycetes. XLIV. New and rare Dematiaceous species from the Transvaal. Mycotaxon 17:301-316.

MOUCHACCA, J. 1990. Champignons de Nouvelle-Calédonie - 1 Quelques dématiées intéressantes de litière forestière. Persoonia 14:151-160.

NAG RAJ, T.R. \& KENDRICK, B. 1975. A monograph of Chalara and allied genera. Wilfrid Laurier University Press, Waterloo.

NAWAWI, A. \& KUTHUBUTHEEN, A.J. 1987. A new species of the genus Subulispora. Mycotaxon 30:459-463.

NAWAWI, A., KUTHUBUTHEEN, A.J. \& SUTTON, B.C. 1990. New species and combinations in Vermiculariopsiella (Hyphomycetes). Mycotaxon 37:173-182.

ONOFRI, S. \& CASTAGNOLA, M. 1983. The genera Ardhachandra and Rhinocladiella, their synonymy. Mycotaxon 18:337-343.

PEERALLY, A. 1991. The classification and phytopathology of Cylindrocladium species. Mycotaxon 40:323-366.

PIROZYNSKI, K.A. 1963. Beltrania and related genera. Mycological Papers 90:1-37.
PIROZYNSKI, K.A. 1968. Cryptophiale, a new genus of Hyphomycetes. Canadian Journal of Botany 46:1123-1127.

PIROZYNSKI, K. A. 1972. Microfungi of Tanzania. Mycological Papers 129:1-64.

PIROZYNSKI, K.A. \& HODGES, JR., C.S. 1973. New Hyphomycetes from South Carolina. Canadian Journal of Botany 51:157-173.

PIROZYNSKI, K.A. \& PATIL, S.D. 1970. Some setose Hyphomycetes of leaf litter in south India. Canadian Journal of Botany 48:567-581.

SCHOENLEIN-CRUSIUS, I.H. \& MILANEZ, A.I. 1998a. Fungos microscópicos da Mata Atlântica de Paranapiacaba, São Paulo, Brasil. Revista Brasileira de Botânica 21:73-79.

SCHOENLEIN-CRUSIUS, I.H. \& MILANEZ, A.I. 1998b. Fungal succession on leaves of Alchornea triplinervia (Spreng.) Muell. Arg. submerged in a stream of an Atlantic Rainforest in the state of São Paulo, Brazil. Revista Brasileira de Botânica 21:253-259.

SUTTON, B.C. 1978. New and interesting Hyphomycetes from Tampa, Florida. Mycologia 70:784-801.

SUTTON, B.C. \& HODGES JR., C.S. 1975. Eucalyptus microfungi: Codinaea and Zanclospora species from Brazil. Nova Hedwigia 26:517-525.

TRUFEM, S.F.B. \& MALATINSZKY, S.M.M. 1995. Fungos micorrízicos arbusculares de Melastomataceae e outras plantas nativas resistentes e sensíveis à poluição na Reserva Biológica do Alto da Serra de Paranapiacaba, SP, Brasil. Hoehnea 22:77-89.

TUBAKI, K. \& YOKOYAMA, T. 1971. Notes on the Japanese Hyphomycetes. V. Transactions of the Mycological Society of Japan 12:18-28. 